https://doi.org/10.15407/dopovidi2019.10.029

УДК УДК 539.3

\title{
В.М. Быстров
}

Институт механики им. С.П. Тимошенко НАН Украины, Киев

E-mail: numer@inmech.kiev.ua

\section{Краевой эффект и приповерхностная потеря устойчивости в слоистом композитном материале при сжатии поверхностной нагрузкой}

Представлено академиком НАН Украины А.Н. Гузем

С использованием основных соотношений трехмерной линеаризированной теории устойчивости в рамках модели кусочно-однородной среды решена задача устойчивости слоистого композитного материала при сжатии армирующих слоев поверхностной нагрузкой. Рассмотрен случай неоднородного докритического состояния, связанного с краевым эффектом в области приложения нагрузки. Использована расчетная модель для граничных условий на боковых сторонах многослойного образиа из композитного материала, которые соответствуют условиям симметрии. Исследовано влияние краевого эффекта на затухание форм приповерхностной потери устойчивости при различных статически эквивалентных нагрузках армирующих слоев композитного материала. Для численного решения задачи применен метод сеток на основе модифищированного вариащионно-разностного подхода.

Ключевые слова: слоистый композитный материал, многослойный представительный элемент, продольное сжатие, поверхностная нагрузка, краевой эффект, приповерхностная потеря устойчивости, трехмерная линеаризированная теория устойчивости, смятие ториов.

Одним из возможных механизмов разрушения однонаправленных композитных материалов (КМ) при сжатии вдоль направления армирования является потеря устойчивости армирующих слоев и волокон. При сжатии однонаправленных КМ поверхностной нагрузкой указанный механизм разрушения может иметь характер приповерхностной потери устойчивости с формами потери устойчивости, затухающими при удалении от поверхности. В $[1,2]$ представлена основанная на трехмерной линеаризированной теории устойчивости деформируемых тел (ТЛТУДТ) [3, 4], континуальная теория разрушения КМ при смятии торцов. Показано, что для однонаправленных КМ (армирующие волокна или слои направлены перпендикулярно к торцу) на первоначальном этапе разрушения КМ при сжатии поверхностной нагрузкой вдоль направления армирования единственно возможным механизмом разрушения является приповерхностная потеря устойчивости в структуре материала, которая предшествует внутренней потере устойчивости. В [5, 6] этот механизм

(C) В.М. Быстров, 2019 
исследован на основе ТЛТУдТ в рамках модели кусочно-однородной среды. В указанных работах предполагалось наличие однородного или кусочно-однородного докритического состояния, а исследования проводились для полупространства или полуплоскости без учета влияния боковых сторон образца из КМ.

Проблема исследования приповерхностной потери устойчивости усложняется при таких условиях нагружения и закрепления элементов конструкций и образцов из КМ, когда в окрестности поверхностной нагрузки возникает неоднородное напряженное состояние (область краевого эффекта). Неоднородность докритического состояния при потере устойчивости в структуре КМ может существенным образом влиять на механизмы разрушения элементов конструкций и образцов из КМ. В [7-9] на основе ТЛТУДТ в рамках модели кусочно-однородной среды предложена расчетная модель для численного решения задачи устойчивости слоистого КМ при одноосном сжатии армирующих слоев поверхностной нагрузкой, когда докритическое состояние является неоднородным. В качестве представительного элемента КМ использована многослойная расчетная область с граничными условиями на боковых сторонах расчетной области, которые соответствуют условиям симметрии. В [9] рассмотрен вопрос о влиянии количества слоев, включенных в представительный элемент КМ, на критические параметры устойчивости КМ. Показано, что при увеличении размера расчетной области вдоль линии приложения поверхностной нагрузки, формы потери устойчивости и зависимость значений критических нагрузок от количества слоев представительного элемента КМ приобретают установившийся характер. В данной работе для численного исследования приповерхностной потери устойчивости слоистого КМ при неоднородном докритическом состоянии использован представительный элемент, включающий девять армирующих слоев. Исследован краевой эффект для докритического состояния и формы приповерхностной потери устойчивости КМ при различных статически эквивалентных нагрузках армирующих слоев композитного материала в широком диапазоне изменения отношения упругих характеристик армирующих слоев и матрицы. Для численного решения задачи применен метод сеток на основе модифицированного вариационно-разностного подхода [10].

Постановка задачи. Рассмотрим задачу определения параметров затухания краевого эффекта для докритического состояния и задачу устойчивости слоистого двухкомпонентного КМ регулярной структуры (рис. 1, $a$ ) для схемы нагружения, которая соответствует одноосному сжатию слоев наполнителя в направлении оси $O x_{2}$ поверхностной нагрузкой постоянной интенсивности $p^{0}$ и в общем случае приводит к неоднородному докритическому состоянию. Эта нагрузка имеет следующий вид:

$$
p\left(x_{1}, 0\right)=\sigma_{22}\left(x_{1}, 0\right)=\left\{\begin{array}{cc}
p^{0}, & -h_{a} / 2+k h \leqslant x_{1} \leqslant h_{a} / 2+k h, \quad k=0, \pm 1, \ldots, \\
0, & \text { для остальных } x_{1} .
\end{array}\right.
$$

Здесь $h=h_{a}+h_{m}$ - параметр структуры слоистого КМ; $h_{a}, h_{m}-$ соответственно толщина слоя наполнителя (армирующего слоя) и слоя связующего (матрицы). Предполагаем, что на бесконечности нагрузка к армирующим слоям прикладывается по такой же схеме. Вдоль оси $O x_{3}$ нагрузка не изменяется. Исходя из условий симметрии нагружения и регулярности структуры КМ, задачу устойчивости решаем для представительного элемента ма- 

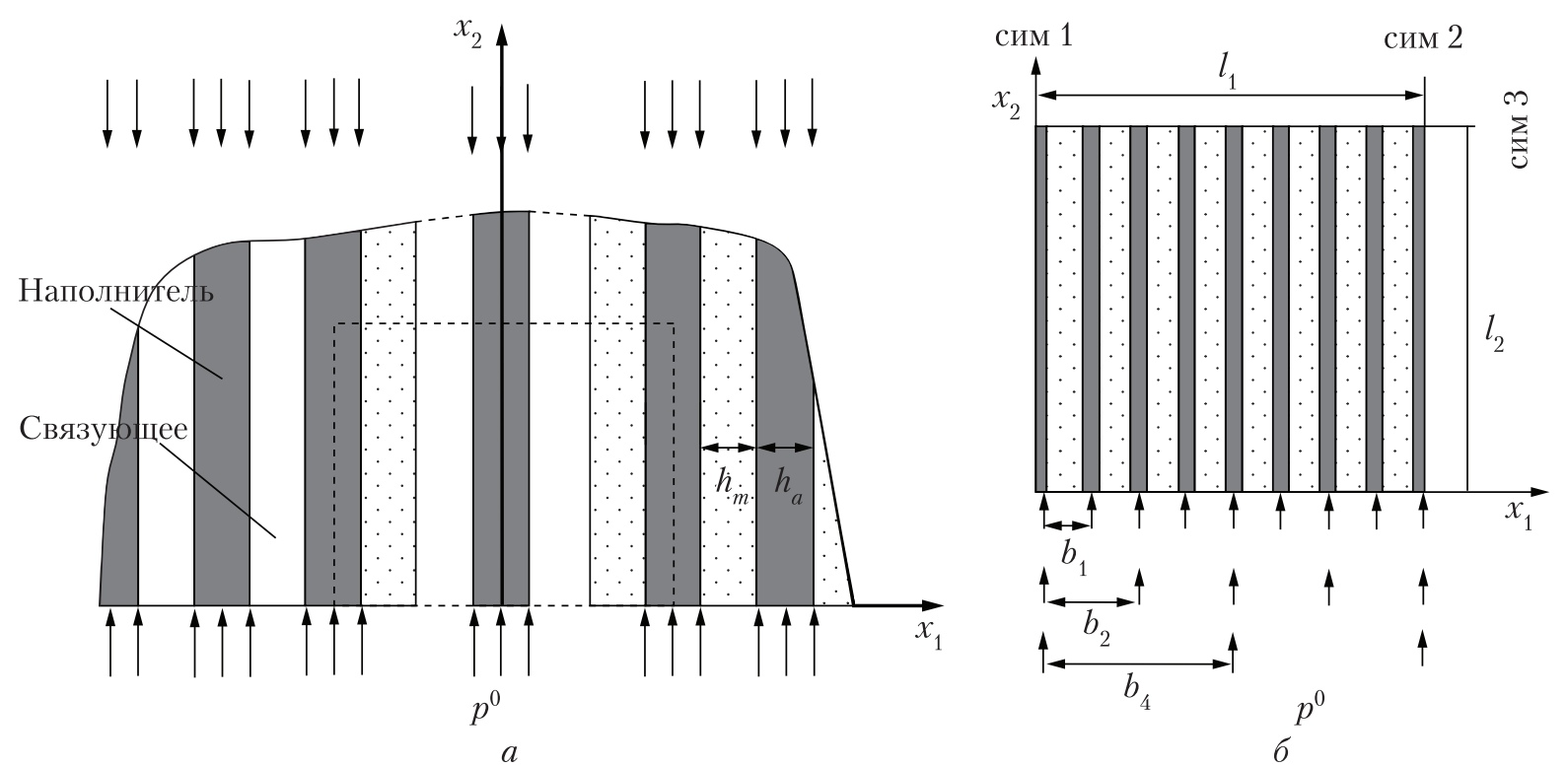

$\boldsymbol{P u c . ~ 1 . ~ С л о и с т ы и ̆ ~ К М ~ ( a ) ; ~ п р е д с т а в и т е л ь н ы и ̆ ~ э л е м е н т ~ м а т е р и а л а ~ ( б ) , ~ с и м ~ 1 , ~ с и м ~ 2 , ~ с и м ~} 3$ - оси симметрии $x_{1}=0, x_{1}=l_{1}, x_{1}=l_{2}$

териала, которому соответствует расчетная область конечных размеров. На основании результатов работы [9] для исследования краевого эффекта и приповерхностной потери устойчивости используется представительный элемент КМ, размер которого в направлении оси $O x_{1}$ составляет $l_{1}=8 h$. Указанная расчетная область показана на рис. 1,6 . Крайние слои представительного элемента являются армирующими слоями. Граничные условия на боковых сторонах расчетной области соответствуют условиям симметрии. Расчеты проводили для различных значений $b_{n}=n h$ периода поверхностной нагрузки в соответствии с последовательной разгрузкой армирующих слоев по следующей схеме: $b_{1}=h, \quad k=0,1, \ldots, 8$; $b_{2}=2 h, k=0,2,4,6,8 ; b_{4}=4 h, k=0,4,8$ (рис 1,6 ). При этом поверхностная нагрузка оставалась статически эквивалентной за счет соответствующего изменения интенсивности $p^{0}$.

Предполагая, что материал наполнителя и связующего является достаточно жестким, при исследовании устойчивости будем применять второй вариант теории малых докритических деформаций $[3,4]$. Поверхностную нагрузку считаем “мертвой”, что обеспечивает выполнение достаточных условий применимости статического метода исследования [3]. При использовании статического метода задача устойчивости сводится к обобщенной задаче на собственные значения, в которой минимальное собственное значение $\mu$ определяет критическую нагрузку, а соответствующая собственная функция $\boldsymbol{u}=\left(u_{1}, u_{2}\right)-$ форму потери устойчивости. Задачу рассматриваем в двумерной постановке для случая плоской деформации в плоскости $x_{1} O x_{2}$.

Начальное состояние определяем из следующих основных соотношений линейной теории упругости:

уравнение равновесия

$$
\sigma_{i j, i}^{0}=0, x \subset \Omega ;
$$


граничные условия

$$
\begin{aligned}
& \sigma_{21}^{0}=0 \wedge u_{2}^{0}=0,0 \leqslant x_{1} \leqslant l_{1} \wedge x_{2}=l_{2} \\
& \sigma_{21}^{0}=0 \wedge u_{1}^{0}=0,\left(x_{1}=0 \vee x_{1}=l_{1}\right) \wedge\left(0 \leqslant x_{2} \leqslant l_{2}\right) \\
& \sigma_{22}^{0}=p \wedge \sigma_{21}^{0}=0,0 \leqslant x_{1} \leqslant l_{1} \wedge x_{2}=0
\end{aligned}
$$

условия идеального контакта между слоями

$$
\left[\sigma_{i 1}^{0}\right]=0, \quad\left[u_{i}^{0}\right]=0 ;
$$

соотношения между компонентами напряжений $\sigma_{i j}^{0}$, деформаций $\varepsilon_{i j}^{0}$ и перемещений $u_{i}^{0}$ в пределах компонента КМ

$$
\sigma_{i j}^{0}=\delta_{i j} A_{i k} \varepsilon_{k k}^{0}+2\left(1-\delta_{i j}\right) G \varepsilon_{i j}^{0}, \varepsilon_{i j}^{0}=\frac{1}{2}\left(u_{i, j}^{0}+u_{j, i}^{0}\right), i \neq j .
$$

где $A_{11}=A_{22}=\frac{E(1-v)}{(1+v)(1-2 v)}, A_{22}=\frac{E v}{(1+v)(1-2 v)}, G=\frac{E}{2(1+v)}$.

Здесь $A_{i j}, E, G, v$ - модули упругости, модуль Юнга, модуль сдвига, коэффициент Пуассона слоя соответственно.

Границу зоны $\Gamma_{\rho}$ и длину затухания $\lambda_{\rho}$ краевого эффекта с точностью $\rho \%$ определяем из следующих соотношений [11]:

$$
\begin{aligned}
& \rho=\left.\tilde{\rho}\left(x_{1}, x_{2}\right)\right|_{x \in \Gamma_{\rho}}, \\
& \lambda_{\rho}=\max _{x_{1}, x_{2} \in \Gamma_{\rho}}\left(x_{2}\right),
\end{aligned}
$$

где функция затухания напряжений $\tilde{\rho}\left(x_{1}, x_{2}\right)$ определяется соотношением

$$
\tilde{\rho}=100\left(\sigma_{22}(x)-\sigma_{y c m}\right) /\left(p\left(x_{1}, 0\right)-\sigma_{y c m}\right) .
$$

Функция $\tilde{\rho}\left(x_{1}, x_{2}\right)$ характеризует изменение нормальных напряжений в зоне краевого эффекта по сравнению со значением самоуравновешенной нагрузки на границе $x_{2}=0$ расчетной области. В (9) $\sigma_{y c m}=\sigma_{22}\left(x_{1}, l_{2}\right)$ - установившееся напряжение на границе $x_{2}=l_{2}$ расчетной области в направлении затухания краевого эффекта.

Основные соотношения трехмерной линеаризированной теории устойчивости для определения критических параметров устойчивости КМ имеют следующий вид:

уравнения в возмущениях

$$
\left(\sigma_{i m}+\mu \sigma_{i k}^{0} u_{m, k}\right)_{, i}=0, \quad x \in \Omega ;
$$

граничные условия 


$$
\begin{aligned}
& \left(\sigma_{12}+\mu \sigma_{22}^{0} u_{1,2}\right)=0 \wedge u_{2}=0,0 \leqslant x_{1} \leqslant l_{1} \wedge x_{2}=l_{2} ; \\
& u_{1}=0 \wedge\left(\sigma_{12}+\mu \sigma_{11}^{0} u_{2,1}\right)=0,\left(x_{1}=0 \vee x_{1}=l_{1}\right) \wedge\left(0 \leqslant x_{2} \leqslant l_{2}\right) \\
& \left(\sigma_{m 2}+\mu p u_{m, 2}\right)=0,0 \leqslant x_{1} \leqslant l_{1} \wedge x_{2}=0 ;
\end{aligned}
$$

условия идеального контакта между слоями

$$
\left[\sigma_{m 1}+\mu \sigma_{1 i}^{0} u_{m, i}\right]=0, \quad\left[u_{i}\right]=0 .
$$

Соотношения между возмущениями напряжений $\delta_{i j}$, деформаций $\varepsilon_{i j}$ и перемещений $u_{i}$ в пределах компонента КМ имеют вид (5), (6).

Критическую нагрузку определяем из следующего соотношения:

$$
p_{\kappa p}=\min |\mu| / l_{1} \int_{0 \leqslant x_{1} \leqslant l_{1}} p\left(x_{1}\right) d x_{1}=\min |\mu| p^{0} h_{a} / h
$$

где $\min |\mu|$ - минимальное по модулю собственное число задачи (10)-(12). Вид соотношения (13) отражает тот факт, что сжимающую нагрузку прикладываем только к армирующим слоям.

Обозначения в (2)-(6), (10)-(12) являются общепринятыми, и индексы изменяются от 1 до 2 ( $\wedge, \vee-$ - знаки логического умножения и сложения). Верхним индексом “0” обозначены компоненты напряжений и перемещений для докритического состояния. Индекс, обозначающий принадлежность к слою КМ, для удобства опущен. В соотношениях $(4),(12)[f(x)]=f(x-0)-f(x+0)-$ скачок функции $f(x)$.

Численное решение. Задачи (2)-(6), (10)-(12) решались методом сеток с использованием концепции базовой схемы [10]. При таком подходе разностные схемы для расчетной области $\Omega$ строятся в каждом сеточном узле как определенная сумма значений базовой схемы, представляющей собой разностную схему, полученную вариационно-разностным способом на шаблоне ячейки разностной сетки. Для решения дискретных задач применяли эффективные численные методы [12] в соответствии с методикой, представленной в работах $[10,13]$. Алгебраическую задачу определения начального состояния решали методом Холецкого, а дискретную задачу устойчивости на собственные значения - методом итерирования подпространства.

Числовые результаты и их анализ. Рассмотрен слоистый композитный материал при сжатии вдоль направления армирования поверхностной нагрузкой, заданной соотношением (1). Расчеты проведены для следующих механических и геометрических характеристик материала: $E_{a} / E_{m}=20,100,500,1000,1500 ; v_{a} / v_{m}=0,3 ; c_{a}=h_{\mu} / h=0,5$. Здесь $E_{a}, E_{m}$ и $v_{a}, v_{m}-$ соответственно, модули Юнга и коэффициенты Пуассона армирующих слоев и слоев связующего, $c_{a}$ - объемное содержание армирующих слоев, $h_{a}-$ толщина армирующего слоя. Размер расчетной области в направлении $O x_{1}$ составил $l_{1}=8 h$. Размер $l_{2}$ расчетной области в направлении $O x_{2}$ определяли в результате вычислительного эксперимента, исходя из условия установившихся значений исследуемых параметров при дальнейшем увеличении размера расчетной области в направлении $O x_{2}$. 

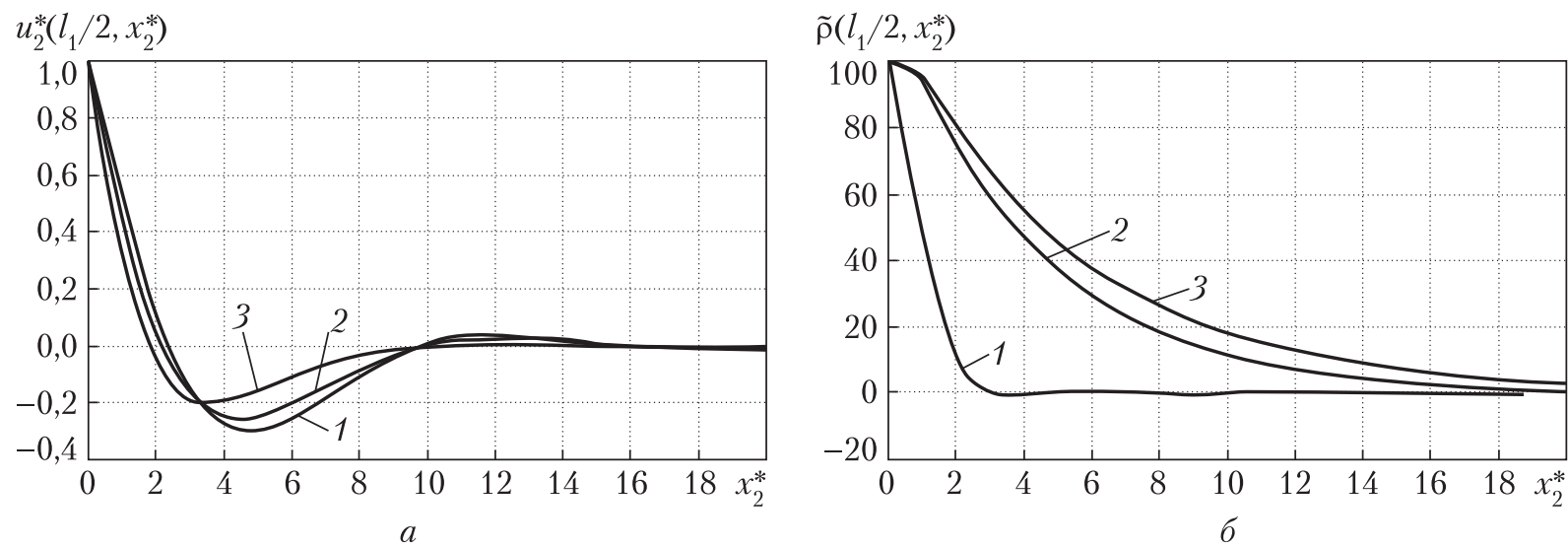

Puc. 2. Формы потери устойчивости $(a)$ и функция затухания напряжений $(\sigma)$ среднего армирующего слоя представительного элемента КМ при $E_{a} / E_{m}=100$ для различных значений $b_{n}=n h$ периода поверхностной нагрузки: $1-h ; 2-2 h ; 3-4 h$

На рис. 2 представлены формы потери устойчивости $u_{2}^{*}\left(l_{1} / 2, x_{2}^{*}\right)=u_{2}\left(l_{1} / 2, x_{2} / h\right) / u_{2}^{\max }$ (рис. $2, a)$ и функции затухания напряжений $\tilde{\rho}\left(l_{1} / 2, x_{2}^{*}\right)=\tilde{\rho}\left(l_{1} / 2, x_{2} / h\right)$ (рис. $\left.2, \sigma\right)$ в сечении $x_{1}=l_{1} / 2$ среднего армирующего слоя представительного элемента КМ, где $u_{2}^{\max }-$ максимальное значение возмущения перемещений в указанном сечении, для различных значений $b_{n}=n h$ периода поверхностной нагрузки. Результаты получены для значения $E_{a} / E_{m}=100-$ отношения модулей Юнга армирующего слоя и матрицы. Качественный характер указанных зависимостей имеет место для всего исследованного диапазона значений $E_{a} / E_{m}$. Как следует из полученных результатов, увеличение периода поверхностной нагрузки, связанного с разгрузкой армирующих слоев, приводит к увеличению зоны краевого эффекта для докритического состояния и увеличению длины полуволны приповерхностной формы потери устойчивости возле нагруженной поверхности. При этом затухание краевого эффекта, соответствующего приповерхностной потери устойчивости, происходит на расстоянии, соизмеримом с длиной волны.

На рис. 3 показана зависимость длины $\lambda_{5 \%}$ затухания краевого эффекта, определенной с точностью $5 \%$ (рис. $3, a)$ и длины $l_{k p}$ волны приповерхностной формы потери устойчивости (рис. 3,6 ) для среднего армирующего слоя представительного элемента КМ от отношения $E_{a} / E_{m}$ модулей упругости армирующего слоя и матрицы для различных значений $b=n h$ периода поверхностной нагрузки. Наблюдается существенная зависимость длины затухания краевого эффекта для докритического состояния от указанного отношения для значений периода нагрузки, превышающих параметр структуры. Для нагрузки, изменяющейся в пределах параметра структуры, увеличение значения отношения $E_{a} / E_{m}$ приводит к локальному перераспределению напряжений в зоне краевого эффекта без увеличения ее размера, который, как видно из рис. 2, б, находится в пределах двух параметров структуры. Длина волны приповерхностной формы потери устойчивости возле нагруженной поверхности растет с увеличением значения $E_{a} / E_{m}$ для всех значений периода нагрузки и возрастает при увеличении количества ненагруженных армирующих слоев.

На рис. 4 показаны формы потери устойчивости (рис. $4, a$ ) и функции затухания напряжений (рис. 4,6$)$ для периода поверхностной нагрузки $2 h$ при изменении отношения $E_{a} / E_{m}$ 

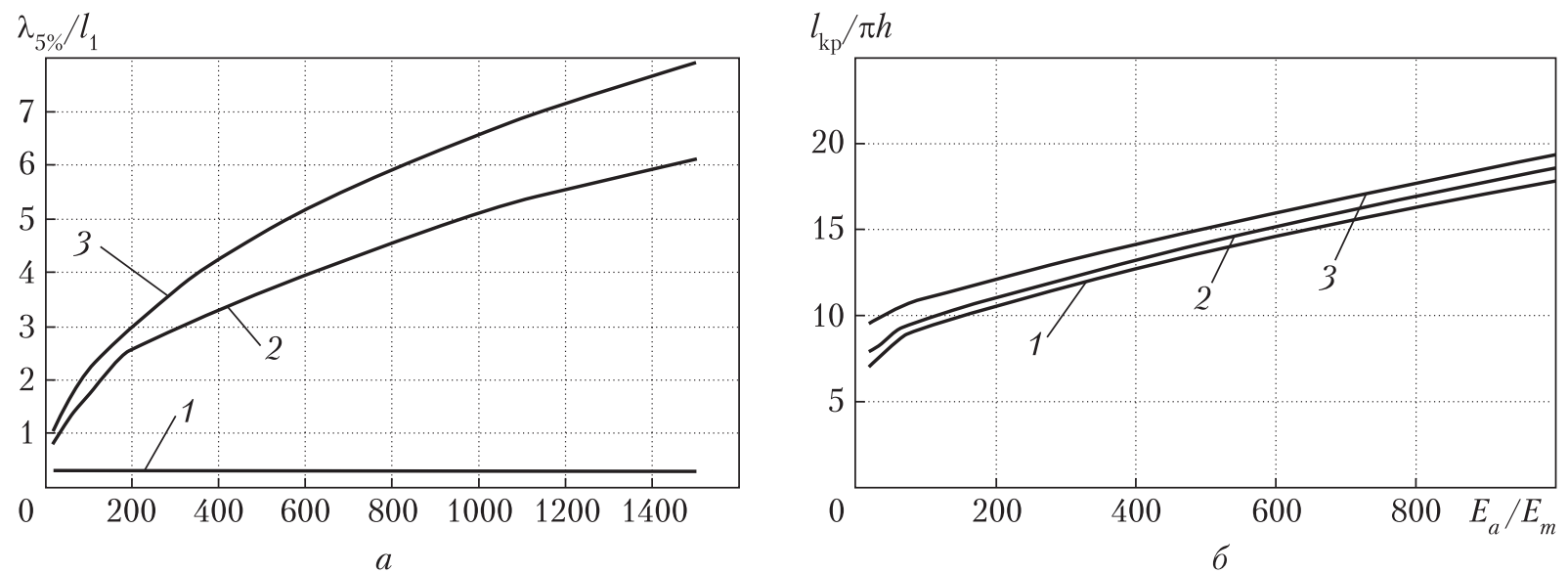

$\boldsymbol{P u c . 3 . ~ З а в и с и м о с т ь ~ д л и н ы ~ з а т у х а н и я ~ к р а е в о г о ~ э ф ф е к т а ~}(a)$ и длины волны формы приповерхностной потери устойчивости (б) среднего армирующего слоя представительного элемента КМ от отношения модулей упругости армирующего слоя и матрицы для различных значений $b_{n}=n h$ периода поверхностной нагрузки: $1-h ; 2-2 h ; 3-4 h$
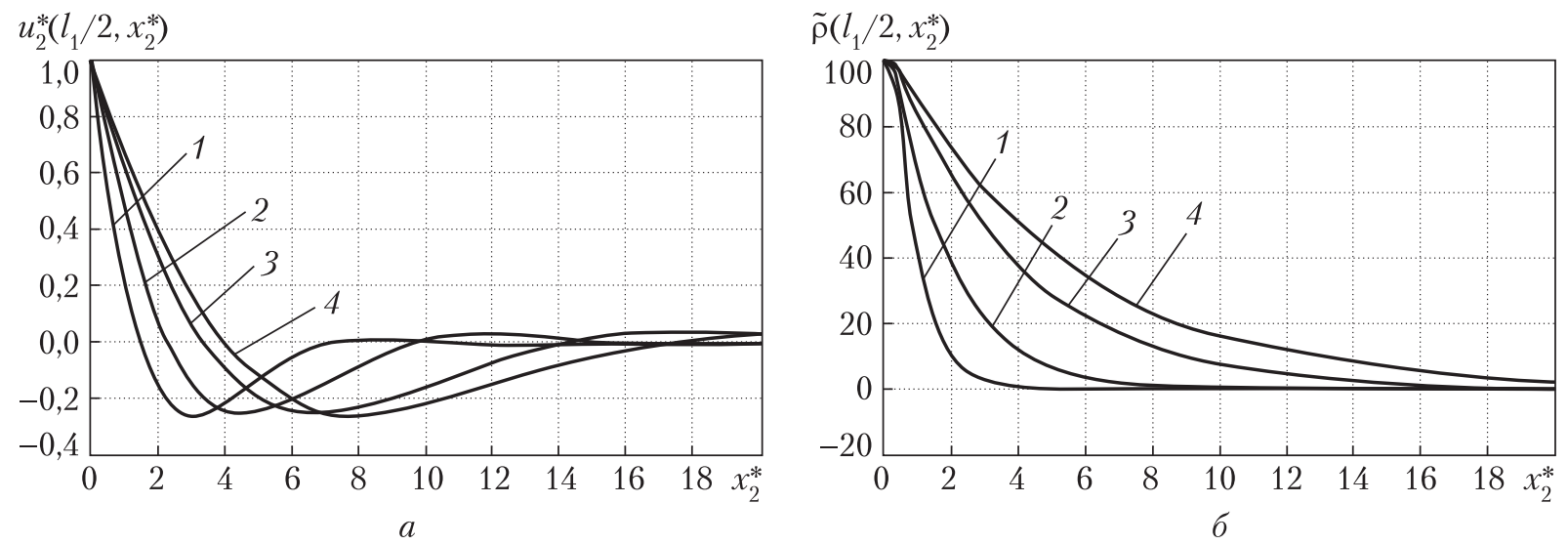

$\boldsymbol{P u c . ~ 4 . ~ Ф о р м ы ~ п о т е р и ~ у с т о и ̆ ч и в о с т и ~ ( ~} a$ ) и функции затухания краевого эффекта $(\sigma)$ для периода поверхностной нагрузки $2 h$ при изменении отношения $E_{a} / E_{m}$ модулей Юнга армирующих слоев и матрицы: $1-20 ; 2-100 ; 3-500 ; 4-1000$

модулей Юнга армирующих слоев и матрицы. Увеличение значения отношения $E_{a} / E_{m}$ приводит к более медленному затуханию краевого эффекта (увеличению зоны краевого эффекта) для докритического состояния и более медленному затуханию форм потери устойчивости.

Полученные результаты позволяют сделать следующие выводы. Расчетная схема на основе ТЛТУдТ и модели кусочно-однородной среды, когда в качестве представительного элемента КМ используется многослойная расчетная область с граничными условиями на боковых сторонах расчетной области, которые соответствует условиям симметрии, позволяет исследовать механизм разрушения при смятии торцов образцов и элементов конструкций из КМ.

Увеличение периода поверхностной нагрузки при разгрузке армирующих слоев обусловливает увеличение зоны краевого эффекта для докритического состояния и, как след- 
ствие, увеличение длины волны приповерхностной потери устойчивости и уменьшение ее амплитуды возле граничной поверхности. Длина затухания краевого эффекта и длина волны приповерхностной формы потери устойчивости возле нагруженной поверхности возрастают с увеличением значения отношения упругих характеристик армирующих слоев и матрицы. Влияние неоднородного докритического состояния, связанного с краевым эффектом, на характер затухания приповерхностных форм потери устойчивости проявляется в большей степени при изменении периода поверхностной нагрузки, чем при изменении соотношения упругих характеристик армирующих слоев и матрицы.

\section{ЦИТИРОВАННАЯ ЛИТЕРАТУРА}

1. Гузь А.Н. Механика разрушения композитных материалов при сжатии. Киев: Наук. думка, 1990. 632 с.

2. Гузь А.Н. Основы механики разрушения композитов при сжатии: В 2-х т. Т. 1. Разрушение в структуре материала. 592 с. Т. 2. Родственные механизмы разрушения. 736 с. Киев: Литера, 2008.

3. Гузь А.Н. Основы трехмерной теории устойчивости деформируемых тел. Киев: Вища школа, 1986. 512 c.

4. Guz A.N. Fundamentals of the Three-Dimensional Theory of Stability of Deformable Bodies. Berlin: Springer, 1999. $555 \mathrm{p}$.

5. Гузь А.Н., Коханенко Ю.В. Хрупкое разрушение композитных материалов при смятии торцов. Докл. AH CCCP. 1987. 296, № 4. C. 805-808.

6. Guz A.N., Kokhanenko Yu.V. Numerical Solution of Three-Dimentional Stability Problems for Elastic Bodies. Int. Appl. Mech. 2001. 37, № 11. P. 1369-1399.

7. Dekret V.A., Zelenskii V.S., Bystrov V.M. Numerical Analysis of Stability of a Laminated Composite with Compressed Reinforcement Plies. Int. Appl. Mech. 2014. 50, № 5. P. 549-557.

8. Bystrov V.M., Dekret V.A., Zelenskii V.S. Loss of Stability in a Composite Laminate Compressed by a Surface Load. Int. Appl. Mech. 2017. 53, № 2. P. 156-163.

9. Быстров В.М., Декрет В.А., Зеленский В.С. Численное исследование устойчивости слоистого композитного материала при сжатии поверхностной нагрузкой. Проблеми обчисл. механіки $i$ мічності конструкиій. 2018. Вип. 28. С. 23-33.

10. Григоренко Я.М., Шевченко Ю.В., Василенко А.Т. и др. Численные методы. Механика композитов: В 12-и т. Под общ. ред. А.Н. Гузя. Т. 11. Киев: А.С.К., 2002. 448 с.

11. Bystrov V.M., Dekret V.A., Zelenskii V.S. Numerical Analysis is of the Edge Effect in a Composite Laminate with Compressed Reinforcement Plies. Int. Appl. Mech. 2015. 51, № 5. P. 561-566.

12. Pissanetzky S. Sparse Matrix Technology. London: Academic Press, 1984. 321 pp.

13. Гузь А.Н., Декрет В.А. Модель коротких волокон в теории устойчивости композитов. Saarbrücken: Lambert Acad. Publ., 2015. 315 c.

Поступило в редакцию 05.07.2019

\section{REFERENCES}

1. Guz, A. N. (1990). Mechanics of fracture of composite materials under compression. Kyiv: Naukova Dumka (in Russian).

2. Guz, A. N. (2008). Fundamentals of the compressive fracture mechanics of composites. Edition in 2 vulumes. Vol. 1. Fracture in structure of materials. Vol. 2. Related mechanisms of fracture. Kyiv: LITERA (in Russian).

3. Guz, A. N. (1986). Fundamentals of three-dimensional theory of stability of deformable bodies. Kyiv: Vysha Shkola (in Russian).

4. Guz, A. N. (1999). Fundamentals of the Three-Dimensional Theory of Stability of Deformable Bodies. Berlin: Springer.

5. Guz, A. N. \& Kokhanenko, Yu. V. (1987). Brittle fracture of composite materials with crushed of ends. Dokl. Acad. nauk S.S.S.R., 296, No. 4, pp. 805-808 (in Russian).

6. Guz, A. N. \& Kokhanenko, Yu. V. (2001). Numerical Solution of Three-Dimentional Stability Problems for Elastic Bodies. Int. Appl. Mech., 37, No. 11, pp. 1369-1399. 
7. Dekret, V. A., Zelenskii, V. S. \& Bystrov, V. M. (2014). Numerical Analysis of Stability of a Laminated Composite with Compressed Reinforcement Plies. Int. Appl. Mech., 50, No. 5, pp. 549-557.

8. Bystrov, V. M., Dekret, V. A. \& Zelenskii, V. S. (2017). Loss of Stability in a Composite Laminate Compressed by a Surface Load. Int. Appl. Mech., 53, No. 2, pp. 156-163.

9. Bystrov, V. M., Dekret, V. A. \& Zelenskii, V. S. (2018). Numerical Study of Stability of Composite Laminate Compressed by a Surface Load. Problems of Computational Mechanics and Strength of Structures, Iss. 28, pp. 23-33 (in Russian).

10. Grygorenko, Ya. M., Shevchenko, Yu. N., Vasilenko, A. T. et al. (2002). Computaional methods. Mechanics of composites: In 12 volumes. Editor-in-Chief A.N. Guz. Vol.11. Kyiv: A.S.K. (in Russian).

11. Bystrov, V. M., Dekret, V. A. \& Zelenskii, V. S. (2015). Numerical Analysis of the Edge Effect in a Composite Laminate with Compressed Reinforcement Plies. Int. Appl. Mech., 51, No. 5, pp. 561-566.

12. Pissanetzky, S. (1984). Sparse Matrix Technology. London: Academ. Press.

13. Guz, A. N. \& Dekret, V. A. (2015). The model of short fibers in the theory of stability of composites. Saarbrücken: Lambert Acad. Publ. (in Russian).

Received 05.07.2019

\section{B.М. Бистров}

Інститут механіки им. С.П. Тимошенка НАН України, Київ

E-mail: numer@inmech.kiev.ua

\section{КРАЙОВИЙ ЕФЕКТ І ПРИПОВЕРХНЕВА ВТРАТА СТІЙКОСТІ У ШАРУВАТОМУ КОМПОЗИТНОМУ МАТЕРІАЛІ ПРИ СТИСКАННІ ПОВЕРХНЕВИМ НАВАНТАЖЕННЯМ}

3 використанням основних співвідношень тривимірної лінеаризованої теорії стійкості у рамках моделі кусково-однорідного середовища отримано розв'язок задачі стійкості шаруватого композитного матеріалу при стисканні поверхневим навантаженням. Розглянуто випадок неоднорідного докритичного стану, пов'язаного з крайовим ефектом в області навантаження. Використана розрахункова модель для граничних умов на бічних сторонах багатошарового зразка з композитного матеріалу, які відповідають умовам симетрії. Досліджено вплив крайового ефекту на згасання форм приповерхневої втрати стійкості при різних статично еквівалентних навантаженнях армуючих шарів композитного матеріалу. Для чисельного розв'язку задачі застосовано метод сіток на основі модифікованого варіаційно-різницевого підходу.

Ключові слова: шаруватий композитний матеріал, багатошаровий представницький елемент, повздожнє стискання, поверхневе навантаження, приповерхнева втрата стійкості, тривимірна лінеаризована теорія стійкості, зминання ториів.

\section{V.M. Bystrov}

S.P. Timoshenko Institute of Mechanics of the NAS of Ukraine, Kyiv

E-mail: numer@inmech.kiev.ua

\section{END EFFECT AND NEAR-SURFACE BUCKLING IN A LAMINATE COMPOSITE MATERIAL COMPRESSED BY A SURFACE LOAD}

Using the basic relations of the three-dimensional linearized theory of stability in the framework of the piecewise-homogeneous medium model, the problem of stability of a layered composite material under compression of reinforcing layers by a surface load is solved. The case of a non-uniform subcritical state associated with an end effect in the region of application of the load is considered. A computational model is used for the boundary conditions on the lateral sides of a multilayer sample made of a composite material, which correspond to the symmetry conditions. The influence of the end effect on the attenuation of forms of a near-surface instability at different statically equivalent loads of reinforcing layers of a composite material is studied. For the numerical solution of the problem, the grid method based on the modified variation-difference approach is applied.

Keywords: layered composite material, multilayer representative element, longitudinal compression, surface load, end effect, near-surface buckling, mode of stability loss, three-dimensional linearized theory of stability, crumpling of ends. 